\title{
Quantum energy inequalities in two dimensions
}

\author{
Christopher J. Fewstel* \\ Department of Mathematics, University of York, Heslington, York, YO10 5DD, UK
}

(Dated: July 24, 2018)

\begin{abstract}
Quantum energy inequalities (QEIs) were established by Flanagan for the massless scalar field on two-dimensional Lorentzian spacetimes globally conformal to Minkowski space. We extend his result to all two-dimensional globally hyperbolic Lorentzian spacetimes and use it to show that flat spacetime QEIs give a good approximation to the curved spacetime results on sampling timescales short in comparison with natural geometric scales. This is relevant to the application of QEIs to constrain exotic spacetime metrics.
\end{abstract}

PACS numbers: 04.62.+v

Keywords: Energy conditions, Quantum inequalities

Classically, the massless (minimally coupled) free scalar field obeys the weak energy condition: it displays a nonnegative energy density to all observers at all points in spacetime. Its quantised sibling is quite different, however, admitting unboundedly negative energy densities at individual spacetime points. Violations of the energy conditions are cause for concern, and a considerable effort has been expended, beginning with the work of Ford [1], in trying to understand what constraints quantum field theory might place on such effects. It turns out that averages of the energy density along, for example, timelike curves obey state-independent lower bounds called quantum inequalities, or quantum energy inequalities (QEIs). In two-dimensional Minkowski space, for example, the massless free field obeys the QEI bound [2]

$$
\int_{\gamma}\left\langle T_{a b}(\gamma(\tau))\right\rangle_{\omega} u^{a} u^{b} G(\tau) d \tau \geq-\frac{1}{24 \pi} \int_{-\infty}^{\infty} \frac{G^{\prime}(\tau)^{2}}{G(\tau)} d \tau
$$

for all Hadamard states $\omega$, where $\gamma$ is the worldline of an inertial observer parametrised by proper time $\tau$ with two-velocity $u^{a}$, and $G$ is any smooth, nonnegative sampling function of compact support [i.e., vanishing outside a compact interval]. The right-hand side is large and negative if $G$ is tightly peaked, but small if it is broadly spread. Thus the magnitude and duration are constrained by a relationship reminiscent of the uncertainty relations: in $d$-dimensional Minkowski space, the energy density can drop below $\rho_{0}<0$ for a time $\tau_{0}$ only if $\left|\rho_{0}\right| \tau_{0}^{d}<\kappa_{d}$ for some (small) constant $\kappa_{d}\left(\kappa_{2}=\pi / 6\right.$, for example).

Many exotic spacetimes (wormholes, warp drives, etc) entail violations of the weak energy condition and it has often been suggested that quantum fields might provide the necessary distributions of stress-energy. Quantum energy inequalities provide a quantitative check on such proposals and have been used to argue that exotic spacetimes are tightly constrained [3, 4]. As no curved spacetime QEIs were available when these references were writ-

*Electronic address: cjf3@york.ac.uk ten, they made use of flat spacetime QEIs, and the validity of their conclusions depends on the assumption that quantum fields in curved spacetimes are subject to the same restrictions as those in flat spacetimes, at least on sampling timescales short in comparison with natural geometric scales. We will refer to this as the 'usual assumption': one of our aims is to establish its general validity in arbitrary two-dimensional globally hyperbolic spacetimes.

Our main tool will be the QEI established for the massless scalar field by Flanagan [5] (based on an earlier argument due to Vollick [6]). As this was originally proved only for those two-dimensional spacetimes which are globally conformal to Minkowski space, we begin by obtaining a generalisation to an arbitrary twodimensional globally hyperbolic spacetime $\left(M, g_{a b}\right)$. The same argument applies to spacetimes with boundaries.

We make use of two observations, the first of which is that any point $p \in M$ has a 'diamond neighbourhood' of the form $D=\operatorname{int}\left(J^{+}(q) \cap J^{-}(r)\right)$ such that $\left(D,\left.g_{a b}\right|_{D}\right)$ (considered as a spacetime in its own right) is globally conformal to the whole of Minkowski space. This may be seen by introducing null coordinates $(u, v)$ in a neighbourhood of $p$ so that the metric takes the form $d s^{2}=e^{2 \sigma} d u d v$ for some smooth function $\sigma$. We may assume, without loss of generality, that this neighbourhood contains a diamond neighbourhood $D$ of $p$ corresponding to coordinate ranges $|u|<u_{0},|v|<v_{0}$, say, and by reparametrising $U=\tan \left(\pi u /\left(2 u_{0}\right)\right), V=\tan \left(\pi v /\left(2 v_{0}\right)\right)$, we see that $\left(D,\left.g_{a b}\right|_{D}\right)$ is conformal to the whole of Minkowski space. Furthermore, we may choose a smooth partition of unity $\chi_{\alpha}$ on $M$ such that each $\chi_{\alpha}$ is supported within some diamond region $D_{\alpha}$ of the above type (and only finitely many $\chi_{\alpha}$ are nonzero at any point of M) [16].

Our second observation is that any state of the field on $\left(M, g_{a b}\right)$ induces a state of the field on each $\left(D_{\alpha},\left.g_{a b}\right|_{D_{\alpha}}\right)$ whose $n$-point functions are simply the restrictions to $D_{\alpha}$ of the $n$-point functions on $M$. Now the renormalised stress-energy tensor at any point $p \in D_{\alpha}$ is obtained from derivatives of the difference between the two-point function and the Hadamard parametrix. Since the latter is determined by the local geometry alone, and therefore 
independent of whether $p$ is thought of as belonging to $M$ or $D_{\alpha}$, it follows that the induced state has the same renormalised stress-energy tensor as that on $\left(M, g_{a b}\right)$.

Combining these two observations, any average of the renormalised stress-energy tensor performed within a compact region of $M$ may be decomposed into a sum of averages performed in finitely many of the $D_{\alpha}$, each of which is subject to the QEIs obtained by Flana- gan [5]. As an example, let $\gamma$ be any smooth timelike curve parametrised by proper time $\tau$, with two-velocity $u^{a}$ and acceleration $a^{a}=u^{b} \nabla_{b} u^{a}$. We follow the conventions of [5] in which $u^{a} u_{a}<0$ for timelike $u^{a}$, and write $\tilde{\chi}_{\alpha}(\tau)=\chi_{\alpha}(\gamma(\tau))$. Then for any Hadamard state $\omega$ of the field on $\left(M, g_{a b}\right)$ and any nonnegative, smooth, compactly supported sampling function $G$, we have

$$
\begin{aligned}
\int_{\gamma}\left\langle T_{a b}(\gamma(\tau))\right\rangle_{\omega} u^{a} u^{b} G(\tau) d \tau & =\sum_{\alpha} \int_{\gamma}\left\langle T_{a b}(\gamma(\tau))\right\rangle_{\omega} u^{a} u^{b} G(\tau) \widetilde{\chi}_{\alpha}(\tau) d \tau \\
& \geq-\frac{1}{24 \pi} \sum_{\alpha} \int_{-\infty}^{\infty}\left[\frac{\left(\widetilde{\chi}_{\alpha} G\right)^{\prime}(\tau)^{2}}{\widetilde{\chi}_{\alpha}(\tau) G(\tau)}+\widetilde{\chi}_{\alpha}(\tau) G(\tau)\left(a^{a} a_{a}+R\right)\right] d \tau \\
& =-\frac{1}{24 \pi} \int_{-\infty}^{\infty}\left[\sum_{\alpha} \frac{\left(\widetilde{\chi}_{\alpha} G\right)^{\prime}(\tau)^{2}}{\widetilde{\chi}_{\alpha}(\tau) G(\tau)}+G(\tau)\left(a^{a} a_{a}+R\right)\right] d \tau
\end{aligned}
$$

where we have used the decomposition mentioned above, and applied the QEI Eq. (1.7) of [5] to the sampling function $\widetilde{\chi}_{\alpha}(\tau) G(\tau)$ in each $D_{\alpha}[17]$. Note that only finitely many of the summands are nonzero, so the right-hand side is finite. In exactly the same way, we may extend other QEIs obtained in [5], which involve averages along spacelike or null curves. The same would apply to spacetime-averaged QEIs of the type considered in [2].

The above argument significantly extends the range of applications of QEIs in two dimensions. In particular, it allows the consideration of the Kruskal extension of the two-dimensional black hole spacetime

$$
d s^{2}=-\frac{32 M^{3} e^{-r /(2 M)}}{r} d U d V
$$

where $r=r(U, V)$ is defined implicitly by

$$
\left(1-\frac{r}{2 M}\right) e^{r /(2 M)}=U V
$$

and $U$ and $V$ are restricted so that $r(U, V)>0$, i.e., $U V<1$. The horizon is located at $r=2 M$, or equivalently $U V=0$, and exterior Schwarzschild is the region $U<0, V>0$. Although not globally conformal to Minkowski space, this spacetime can be covered by diamond neighbourhoods, each of which is globally conformal to Minkowski space. The only geometrical constraint on these neighbourhoods is that they should remain inside the physical region $U V<1$. The QEI bounds will become quite weak for trajectories which approach the singularity, owing to the divergence of the Ricci scalar $R=4 M / r^{3}$. As noted by Flanagan [5], QEIs along worldlines which remain close to, but outside, the horizon will also be rather weak due to their large acceleration. For example, a worldline with constant $r>2 M$ has $a^{a} a_{a}=M^{2} /\left(r^{3}(r-2 M)\right)$. However, as Roman has emphasised 7] (and pace 8]) this does not mean that there are no QEI constraints near the horizon [18]. Indeed, in the case $M \gg 1$, it is clear that QEIs along the worldline of a freely falling observer passing through the horizon differ little from the flat space results for averages near the horizon (note $R=1 /\left(2 M^{2}\right.$ ) at $r=2 M$ ). Of course the averaging must be completed well before the singularity is reached, which sets an upper limit on the proper time available. For further discussion of these issues see [5, 6, 7].

We are now in a position to prove the validity of the usual assumption. Since we are concerned with averaging over small scales, it suffices to consider a single diamond region $D$ which is globally conformal to Minkowski space. Suppose a smooth timelike curve $\gamma$ in $D$ may be parametrised by proper time in the interval $|\tau|<T$, say. Then Eq. (2) reduces to

$$
\begin{aligned}
& \int_{\gamma}\left\langle T_{a b}(\gamma(\tau))\right\rangle_{\omega} u^{a} u^{b} G(\tau) d \tau \\
& \quad \geq-\frac{1}{24 \pi} \int_{-\infty}^{\infty}\left[\frac{G^{\prime}(\tau)^{2}}{G(\tau)}+G(\tau)\left(a^{a} a_{a}+R\right)\right] d \tau
\end{aligned}
$$

for any smooth nonnegative 'sampling function' $G$ with compact support in $(-T, T)$.

We note that the QEI bound consists of two parts: the flat spacetime result, and correction terms due to the acceleration of the curve and the scalar curvature of spacetime. As we now show, the first part will dominate if $G$ is peaked on scales short in comparison with those set by $R$ and $a^{a}$. Indeed, putting

$$
A=\sup _{\gamma} a^{a} a_{a} \quad \text { and } \quad B=\max \left\{0, \sup _{\gamma} R\right\},
$$

and replacing $G$ by the scaled version $G_{\tau_{0}}$ defined by

$$
G_{\tau_{0}}(\tau)=\tau_{0}^{-1} G\left(\tau / \tau_{0}\right)
$$


Eq. (15) implies that

$$
\int_{\gamma}\left\langle T_{a b}(\gamma(\tau))\right\rangle_{\omega} u^{a} u^{b} G_{\tau_{0}}(\tau) d \tau \geq-\frac{A+B}{24 \pi}-\frac{C}{24 \pi \tau_{0}^{2}},
$$

where the constant $C$ is given in terms of the 'unscaled' sampling function as

$$
C=\int_{-\infty}^{\infty} G^{\prime}(\tau)^{2} / G(\tau) d \tau
$$

(We have also used the fact that $a^{a} a_{a} \geq 0$.) It is easy to find examples of $G$ supported within an interval of unit proper time with $C$ of the order of 40 (the minimum value is $4 \pi^{2}[19]$ ). Accordingly, if

$$
\tau_{0} \lesssim 10^{-3} \min \left\{A^{-1 / 2}, B^{-1 / 2}\right\}
$$

and $\tau_{0}<2 T$ (i.e., sampling occurs within $D$ ) the second term in Eq. (8) dominates over the first by a factor of around 10 and the flat space result may be safely utilised, certainly for the order-of-magnitude considerations required in [3, 4].

We have therefore justified the usual assumption for the massless scalar field in two-dimensions. Three geometric scales are relevant: the acceleration of the observer, the scalar curvature, and the maximum size (as measured by $T$ ) of diamond neighbourhood globally conformal to the whole of Minkowski space. The last of these becomes relevant when the spacetime contains boundaries or singularities (cf. $[9]$ ).

To conclude, let us consider the status of the usual assumption for dimensions other than two and/or massive fields. Since refs. [3, 4] were written, QEIs have been established in curved spacetimes and such results exist for the free scalar 5, 10, 11, 12], Dirac [6, 13], Maxwell and Proca fields 14, 15] in various levels of generality, including very general results. With the exception of [5, [6] (and those discussed above), these bounds have been "difference" QEIs: namely, the quantity bounded is the difference between the energy density in the state of interest and that in a reference state. For example, in [12] a QEI was obtained for the scalar field in an arbitrary globally hyperbolic spacetime $\left(M, g_{a b}\right)$ for sampling along any smooth timelike curve $\gamma$, which took the form

$$
\begin{array}{r}
\int_{\gamma}\left[\left\langle T_{a b}(\gamma(\tau))\right\rangle_{\omega}-\left\langle T_{a b}(\gamma(\tau))\right\rangle_{\omega_{0}}\right] u^{a} u^{b} G(\tau) d \tau \\
\geq-\mathcal{Q}\left[M, g_{a b}, \gamma, \omega_{0}, G\right]
\end{array}
$$

where $\omega_{0}$ is an (arbitrary, but fixed) reference Hadamard state. This bound holds for arbitrary Hadamard states $\omega$ and any $G$ of the form $G(\tau)=g(\tau)^{2}$ with $g$ real-valued, smooth and compactly supported; an explicit formula for $\mathcal{Q}$ can be given 12]. Now it is easy to see that an "absolute" QEI follows immediately, simply by correcting the "difference" bound by the renormalised energy density of the reference state:

$$
\begin{aligned}
\int_{\gamma}\left\langle T_{a b}(\gamma(\tau))\right\rangle_{\omega} & u^{a} u^{b} G(\tau) d \tau \\
\geq & -\mathcal{Q}\left[M, g_{a b}, \gamma, \omega_{0}, G\right] \\
& +\int_{\gamma}\left\langle T_{a b}(\gamma(\tau))\right\rangle_{\omega_{0}} u^{a} u^{b} G(\tau) d \tau
\end{aligned}
$$

Of course, the problem is that these expressions depend on the reference state, and in a general spacetime it is not usually possible to write down a closed form expression for the stress-tensor of any particular Hadamard state. But now replace $G$ by its scaled version defined by Eq. (7). The difference QEI bound is expected to scale as $\tau_{0}^{-d}$ in $d$-dimensions, and to approach the corresponding Minkowski space bound for sufficiently small $\tau_{0}$. This indeed occurs in examples [11] and a general proof is probably not too difficult. On the other hand, the second term will approach the constant value $\left\langle T_{a b}(\gamma(0))\right\rangle_{\omega_{0}} u^{a} u^{b}$ as $\tau_{0} \rightarrow 0$ and is therefore swamped by the first term when $\tau_{0}$ is small enough.

To establish the usual assumption we would need to quantify how small is 'small enough'. In examples, the difference QEI approaches the corresponding Minkowski results on timescales short in comparison with geometric scales, but there remains the problem of the constant term arising from the reference state. It has not (yet) been ruled out that the reference state could make an anomalously large contribution [20] in which case the timescale $\tau_{0}$ might have to be chosen very much shorter than natural geometric scales. In this case the QEI bound would be very weak, and perhaps insufficient to constrain the geometry as in [3, 4]. At present there is, therefore, a small gap in justifying the usual assumption in dimensions greater than two. However, the results presented here strengthen the expectation that it can be bridged.

\section{Acknowledgments}

I thank Éanna Flanagan and Tom Roman for useful comments.
[1] L.H. Ford, Proc. R. Soc. Lond. A 364, (1978) 227.

[2] É.É. Flanagan, Phys. Rev. D 56, (1997) 4922.
[3] L.H. Ford and T.A. Roman, Phys. Rev. D 53, (1996) 5496. 
[4] M.J. Pfenning and L.H. Ford, Class. Quantum Grav. 14, (1997) 1743.

[5] É.É. Flanagan, Phys. Rev. D 66, (2002) 104007.

[6] D.N. Vollick, Phys. Rev. D 61, (2000) 084022.

[7] T.A. Roman, 'Some thoughts on energy conditions and wormholes', to appear in the Proceedings of the Tenth Marcel Grossmann Meeting on General Relativity and Gravitation, gr-qc/0409090

[8] S.A. Hayward, Int. J. Mod. Phys. D8, (1999) 373.

[9] L.H. Ford, M.J. Pfenning, and T.A. Roman, Phys. Rev. D 57, (1998) 4839.

[10] M.J. Pfenning and L.H. Ford, Phys. Rev. D 57, (1998) 3489.

[11] C.J. Fewster and E. Teo, Phys. Rev. D59, (1999) 104016.

[12] C.J. Fewster, Class. Quantum Grav. 17, (2000) 1897.

[13] C.J. Fewster and R. Verch, Commun. Math. Phys. 225, (2002) 331.

[14] M.J. Pfenning, Phys. Rev. D 65, (2002) 024009.

[15] C.J. Fewster and M.J. Pfenning, J. Math. Phys. 44, (2003) 4480.

[16] The existence of such a partition of unity follows from a paracompactness argument: first cover $M$ by open diamond regions each of which has compact closure and is globally conformal to Minkowski space. By paracompactness, there is a locally finite refinement $\mathcal{O}_{\alpha}$ of this cover, so that each $\mathcal{O}_{\alpha}$ is contained in one of the original diamond regions and therefore has compact closure. We then choose $\chi_{\alpha}$ to be a subordinate partition of unity.

[17] The integrand on the right-hand side of Eq. (5) is taken to vanish outside the support of $G$. To convert Eq. (5) to a signature in which $u^{a} u_{a}>0$ for timelike $u^{a}$, it is necessary only to reverse the sign of $a^{a} a_{a}$.

[18] As is evident from Eq. (2), QEIs along highly accelerated trajectories give weak bounds even in Minkowski space.

[19] Set $G(\tau)=2 \sin ^{2}(\tau \pi)$ for $0<\tau<1$ and zero elsewhere, which attains $C=4 \pi^{2}$. Although this function is only $C^{1}$, there exist smooth $G$ with values of $C$ arbitrarily close to this value.

[20] In order to invalidate the usual assumption, however, it would be necessary to show that all Hadamard reference states gave such an anomalous contribution. 